\title{
Slow dynamics in a turbulent von Kármán swirling flow
}

\author{
A. de la Torre 1 , and J. Burguete 1 ,丹 \\ ${ }^{1}$ Departamento de Física y Matemática Aplicada \\ Universidad de Navarra, P.O.Box 177, E-31080 Pamplona, Spain
}

\begin{abstract}
We present an experimental study of a turbulent von Kármán flow produced in a cylindrical container using two propellers. The mean flow is stationary up to $R e=10^{4}$, where a bifurcation takes place. The new regime breaks some symmetries of the problem, and is time-dependent. The axisymmetry is broken by the presence of equatorial vortices with a precession movement, being the velocity of the vortices proportional to the Reynolds number. The reflection symmetry through the equatorial plane is broken, and the shear layer of the mean flow appears displaced from the equator. These two facts appear simultaneously. In the exact counterrotating case, a bistable regime appears between both mirrored solutions and spontaneous reversals of the azimuthal velocity are registered. This evolution can be explained using a three-well potential model with additive noise. A regime of forced periodic response is observed when a very weak input signal is applied.
\end{abstract}

PACS numbers: 05.45.-a, 47.27.-i, 47.32.-y, 47.65.-d

Introduction - Turbulent flows are ubiquitous in nature: ranging from small scales (heart valves, turbulent mixing) to very large scales (clouds, tornadoes, oceans, earth mantle, the sun and other astrophysical problems), turbulence is present in many applied and fundamental physical problems [1]. But, in spite of the attention it has received, there are still many open questions: the emergence of coherent structures, as vortices, in fully developed turbulence or the rise of different bifurcations on the mean flow [2].

Here we will analyze a particular configuration, the von Kármán swirling flow, where two different propellers are rotating inside a cylindrical cavity. These flows have been

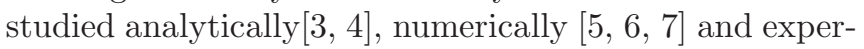
imentally [8, 9, 10]. Recently it has been shown that they can present multistability and memory effects 11]. Thus, this configuration is a natural candidate to study the instabilities that appear in fully developed turbulence and the role played by symmetry breaking and coherent structures. They have been also used in magnetohydrodynamics (MHD) experiments looking for the dynamo action with recent successful results 12] and with a very rich dynamics of the magnetic field [13]. Because of turbulence, the whole MHD problem can not be studied numerically: a usual approach is to deal with stationary mean flows in the so called kinematic dynamo scheme. Although these numerical studies have been used to predict thresholds of the dynamo action [14, 15, 16], real flows can present slow dynamics compared to the magnetic diffusion time that can not be neglected and should be taken into account in the numerical codes.

In this paper we will focus on the slow dynamics that appear for very large $R e$ numbers. The flow presents a non-trivial alternation between two states that break symmetries of the problem. This dynamics, which can be assimilated to a Langevin system with a classical exponential escape time and forced periodic response.

Experimental setup - The experimental volume (Fig 1) is a closed, horizontal cylinder whose diameter $D=2 R=$ $20 \mathrm{~cm}$ is fixed while the height $H$ can be modified continuously. Two propellers are placed at both ends, with radius $R_{\text {prop }}=8.75 \mathrm{~cm}$ and 10 curved blades, each blade with a height of $2 \mathrm{~cm}$ and a curvature radius of $5.0 \mathrm{~cm}$. Using the standard cylindrical coordinate system, the north $N$ (resp. south $S$ ) propeller placed at $z=H / 2$ (resp. $z=-H / 2$ ) has negative (resp. positive) azimuthal velocity. The propellers are impelled by two indepen-

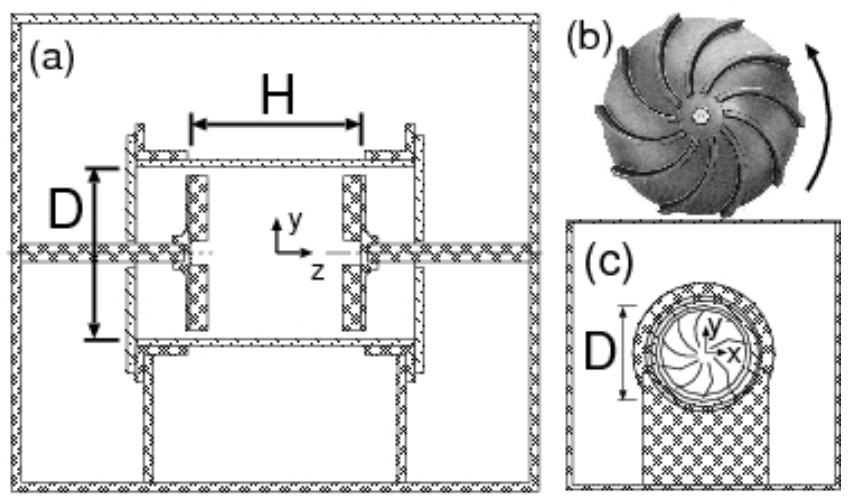

FIG. 1: Experimental setup. (a) Horizontal cylinder with the propellers, inside the tank. The north propeller is at $z=H / 2$ and the south is at $z=-H / 2$. (b) Photograph of the propeller. (c) Scheme of the south propeller viewed from the equatorial plane. The rotation sense with the convex side sets the azimuthal velocity as positive.

dent motors of $3 \mathrm{~kW}$ total power, allowing a rotation frequency in the range $f=0-20 \mathrm{~Hz}$. The frequency of the motors is controlled with a waveform function generator and a PID servo loop control. The cylinder is placed inside a tank of $150 \mathrm{l}$ of volume in order to avoid optical problems and to assure the temperature stability. The fluid used is water at $21^{\circ} \mathrm{C}$.

The measurement of the velocity field is performed using a LDV system (with a chosen spatial resolution of $1 \mathrm{~cm}$ and temporal resolution up to $100 \mathrm{kHz}$ ) and a PIV system (spatial resolution of $1 \mathrm{~mm}$ and temporal resolution of $15 \mathrm{~Hz}$ ). The LDV system allows the measurement of two components of the velocity field (axial $v_{z}$ and azimuthal $v_{\theta}$ ), while the radial component $\left(v_{r}\right)$ is obtained by mass conservation. The velocity field ob- 

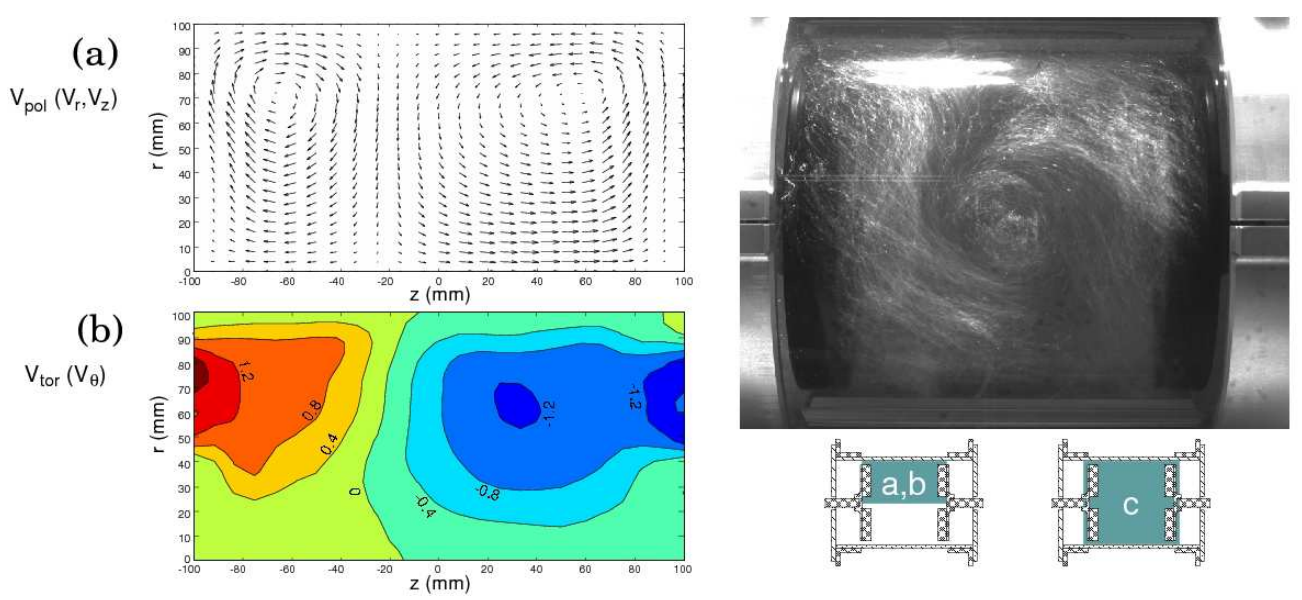

(c)

FIG. 2: (Color online) (a) Stream-vectors $V_{r}, V_{z}$ in the plane $\theta=0$. (b) Contourplot of $V_{\theta}$. The north propeller is on the right, the south propeller on the left. (c) Vortex visualized with air bubbles. (d) Gray zones indicating the regions where the figures $(\mathrm{a}, \mathrm{b}, \mathrm{c})$ were obtained in the cell.

tained in this way is consistent with the PIV measurements in an axial, horizontal plane $(\theta=0)$. Both techniques are based on the displacement of small particles $\left(14 \mu \mathrm{m}, \rho=1.65 \mathrm{~g} / \mathrm{cm}^{3}\right)$ inside the bulk of the fluid.

In our experimental setup three parameters can be modified. The first one is the Reynolds number $R e=$ $R V_{\text {prop }} / \nu$, defined using the propeller's rim velocity $V_{\text {prop }}=2 \pi R_{\text {prop }} f$. This number can be varied continuously in the range $R e \sim 10^{3}-10^{6}$. The second one is the aspect ratio of the experimental volume, $\Gamma=H / D$, set to 1 in this experiment. The last one is the frequency disymmetry, defined as $\Delta=\left(f_{N}-f_{S}\right) /\left(f_{N}+f_{S}\right)$, where $f_{N}$ and $f_{S}$ are the frequencies of each propeller, north and south. This parameter is varied in the range $|\Delta|<0.1$.

Results - For the Re range explored, the flow is in fully-developed turbulence regime. The mean flow $V=$ $\langle v\rangle=\left(V_{r}, V_{\theta}, V_{z}\right)$ represented in Fig[2(a,b) is obtained with the LDV system, averaging velocity series longer than 300 times the period of the propeller. The measurement is done in the plane $\theta=0$ with $R e=310^{5}$ and $\Delta=0$. The flow is divided into two toroidal cells, each of them following its propeller (positive azimuthal velocity in the south, negative in the north). In each cell, the flow is aspired trough the axis towards the propellers, where it is ejected to the walls. The flow then returns along the cylinder's wall, approaching the axis near the equatorial plane. Using PIV measurements, the instantaneous field can be obtained, and no traces of the mean flow are observed. This is due to the high turbulence rate ( $r m s$ value over the mean value) which vary between $60-150 \%$, depending on the spatial position and the velocity component measured.

This mean flow does not preserve the symmetry around the equator (a $\pi$ rotation around any axis in the $z=$ 0 plane, i.e. $R_{\pi}$ symmetry). In the case presented in figure 2(a,b) the cell near the north propeller is bigger than the south one. The broken symmetry is recovered when the mirror state (south cell bigger than the north cell) is considered. Each state (labeled as 'north' $N$ or 'south' $S$ depending on which cell is the dominant one, i.e. bigger) are equally accessible when the system starts from rest. This disymmetry is in contradiction with other works [8] in which the $R_{\pi}$ symmetry is observed (i.e., the frontier between the two rolls is always in the plane $z=$ 0 ), probably due to the presence of baffles or inner rings, which would enforce this condition. Other experiments at much slower Reynolds numbers 10] (Re $\leq 600)$ have shown that the axisymmetry can be broken producing near heteroclinic orbits, but preserving the $R_{\pi}$ symmetry.

When larger tracers (air bubbles) are used, coherent structures as vortices (Fig 2r) are visualized. These structures mostly relay in the dominant cell, so the azimuthal velocity of the vortices are directly related with the azimuthal velocity of the dominant cell. These vortices have a characteristic size $D_{\text {vortex }}=5 \mathrm{~cm}$ and they appear simultaneously to the disymmetry of the mean flow. Previous works in similar configurations [7] showed the formation of a static vortex in the equatorial plane $(z=0)$ for much lower $R e$, inaccessible with the present configuration.

The state $N$ or $S$ of the system can be characterized using different variables. One is the position of the frontier $z_{0}$, defined as the $z$ position in where $V_{\theta}=0$ (in Fig $2 \mathrm{~b}, z_{0} \sim-20 \mathrm{~mm}$ ). Another possibility is measuring $V_{\theta}^{e q}$, the mean azimuthal velocity at an equatorial point near the wall $(r=0.9 R, z=0)$ with the LDV system (in Fig[2 $\left.b, V_{\theta}^{e q} \sim-0.4 \mathrm{~m} / \mathrm{s}\right)$. This mean velocity is stable in time and proportional to the propeller's rim velocity. For $R e<10^{4}$ we find that the normalized azimuthal velocity $\left(U_{\theta}=V_{\theta}^{e q} / V_{\text {prop }}\right)$ is nearly null so the mean flow is almost symmetric. As the $R e$ is increased the disymmetry becomes more notorious, until a plateau ( $U_{\theta}=$ const) is reached for $R e>10^{5}$.

For this range of $R e$ the system can spontaneously jump from one state to the other (inversions). In Fig](a) the instantaneous azimuthal velocity of the equator is plotted versus time $\left(t_{a c q}=510^{4} \mathrm{~s} \sim 14 \mathrm{~h}\right)$ for $R e=$ $2.710^{5}, \Delta=0$. The typical transition time is about $10 \mathrm{~s}$, 

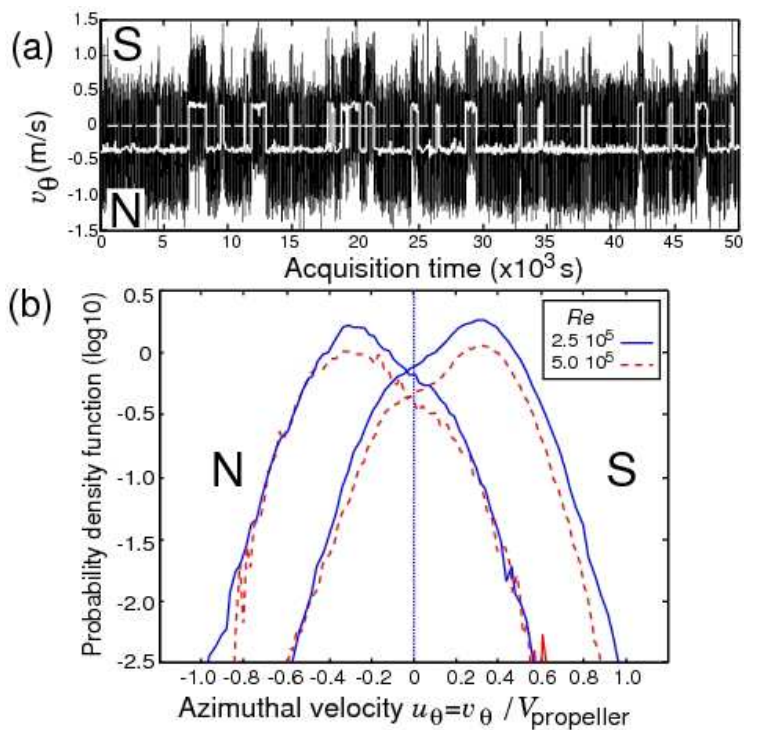

FIG. 3: (a) Inversions of the azimuthal velocity at a point near the wall in $z=0$ for $\Delta=0, R e=2.510^{5}$. Solid white line: filtered data using a low-pass filter with a cutoff frequency $f_{\text {cut }}=0.025 \mathrm{~Hz}$.(b) PDF of $u_{\theta}$ for each state $(N$ or $S$ ) obtained for $\Delta=0$ and various $R e$.

while the time between inversions can vary from minutes to hours. These time scales are much slower than the period of the propeller $\left(t_{a c q}=2.410^{5} T_{\text {prop }}\right.$, with $T_{\text {prop }}=1 / f_{\text {prop }}=0.2 \mathrm{~s}$ ).

The probability density function (PDF) of these states can be computed breaking up the data series in time intervals: a low pass filter is applied (fig 3) (a), white line) that allows to differentiate between $N$ and $S$ and split the signal. The shape of these PDFs does not depend on the cutoff frequency except for extreme values $\left(f_{\text {cut }} \rightarrow 0\right.$ or $f_{\text {cut }} \rightarrow$ sampling rate). Fig 3 (b) shows the $\mathrm{PDF}$ of the normalized instantaneous azimuthal velocity $\left(u_{\theta}=v_{\theta} / V_{\text {prop }}\right)$ for the two states: north (with negative most probable $u_{\theta}$ ) and south (with positive most probable velocity). Each distribution $\left(p_{N}\right.$ or $\left.p_{S}\right)$ is described as the superposition of two gaussians:

$$
\begin{aligned}
p_{N, S}\left(u_{\theta}\right) & =G_{0}+G_{N, S}=\frac{A_{0}}{\sqrt{2 \pi} \sigma_{0}} \exp \left(-\frac{u_{\theta}^{2}}{2 \sigma_{0}^{2}}\right) \\
& +\frac{A_{N, S}}{\sqrt{2 \pi} \sigma_{N, S}} \exp \left(-\frac{\left(u_{\theta}-u_{N, S}\right)^{2}}{2 \sigma_{N, S}^{2}}\right)
\end{aligned}
$$

with $A_{0}+A_{N, S}=1$.

Both distributions $p_{N}$ and $p_{S}$ share the same properties: one of the gaussians $G_{0}$ has zero mean, while the other $G_{N, S}$ is centered around a finite value $\left(\left|u_{N}\right|=\right.$ $\left|u_{S}\right| \neq 0$ ) that increases slightly with the $R e$ number. The amplitudes $A_{0}$ and $A_{N, S}$ have a stronger dependency on the $R e$ (Fig 4 ). For low $R e$ the PDF are nearly gaussians $\left(A_{0} \simeq 1\right)$ while for large $R e$ (plateau) the nonsymmetric gaussian becomes dominant $A_{N, S} \gg A_{0} \neq 0$. The zero mean gaussian $G_{0}$ is due to residues of the symmetric flow and the other gaussian $G_{N, S}$ is related to the displacement of the vortices around the equator.
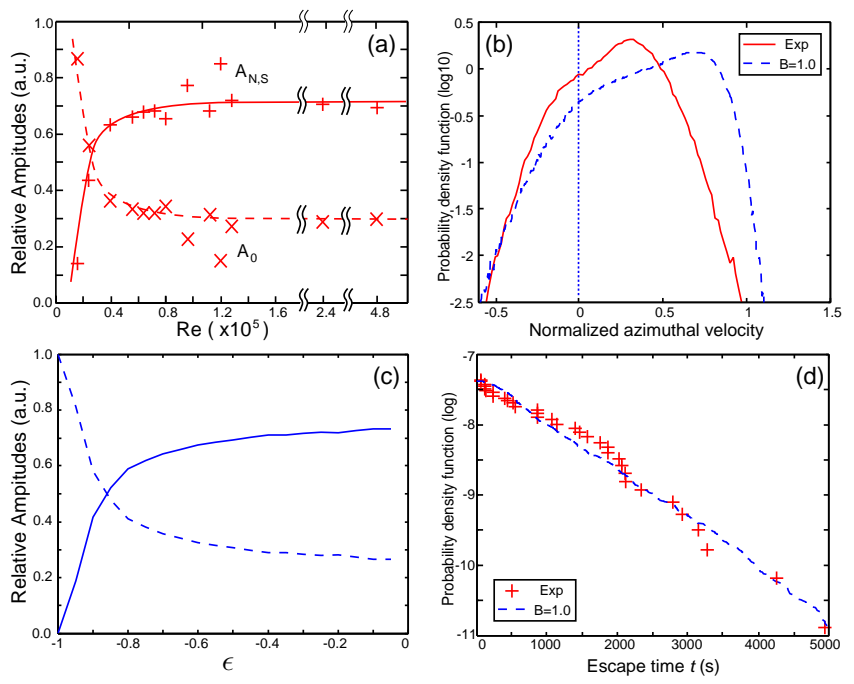

FIG. 4: (Color online) (a) Experimental amplitude of the gaussians $A_{0}\left(\times,-^{-}\right)$and $A_{N, S}(+,-)$ in Eq1 $v s$. Re. The lines are only plotted to indicate the trend and are not obtained from any fitting. (b) PDF of $u_{\theta}$ for the $S$ state for the experimental data series $R e=2.510^{5}$ of Fig 3 (solid line) and numerical data (dashed line) using the model (eq2) with $\epsilon=-0.05, g=2, B=1$. (c) Numerical amplitudes of the $u_{0}(-)$ and $u_{N, S}(--) v s . \epsilon$. Each point corresponds to an ensemble average of 100 realizations. The noise intensity increases linearly with $\epsilon$, in such a way that $B=0$ (resp. 1) when $\epsilon=-1$ (resp. -0.05). (d) PDF of the escape times for experimental data of Fig. $3(+)$ and for the numerical data presented in (b) (dashed line, time unit $\tau_{\text {sim }}=391 \mathrm{~s}$ ).

According to this description, the system visit three different regions in phase space around $u_{\theta}=\left[0, u_{N}, u_{S}\right]$. A simple model based on a three well potential (one for the symmetric case $u_{\theta}=0$ and the two others for the asymmetric states $u_{N, S}$ ) will describe this dynamics:

$$
\dot{u}_{\theta}=\epsilon u_{\theta}+g u_{\theta}^{3}-u_{\theta}^{5}+\sqrt{2 B} \xi(t)
$$

where $\xi(t)$ is a noise distribution with noise level $B$ (playing the role of the turbulence rate) and $g$ controls the relative depth of the potential wells. Thus, the $N$ state (resp. $S$ ) will appear when the system is wandering between the wells $u_{0}$ and $u_{N}$ (resp. $u_{0}$ and $\left.u_{S}\right)$. The parameter $\epsilon$ is varied in the range $\left(-g^{2} / 4<\epsilon<0\right)$ where the three solutions $u_{\theta}=\left[0, \pm\left(g / 2+\left(g^{2} / 4+\epsilon\right)^{0.5}\right)^{0.5}\right]$ are stable.

Different runs using an Euler-Maruyama scheme [17] were performed in order to recover the dynamics: for small $\epsilon$ the dynamics is confined to the region around $u_{\theta}=0$ whereas for $\epsilon \rightarrow 0$ the numerical evolution presents spontaneous inversions. In this later case the PDF of each state can be computed and compared to experiments (fig. 4.b). The characteristic doubly bumped distribution is obtained, but in the numerical distribution the queues are not symmetric due to the shape of the 6 -th order potential in the neighborhood of $u_{S}$. The relative weight of each one of the solutions in the numerical PDFs can be calculated (fig. 4.c) and compared with the experimental amplitudes of fig. 4. a. 
The distribution of the times the system stays in one state (residence times) follows an exponential decay law (Kramer's escape rate [18, 19, 20]):

$$
\rho(t)=1 / T_{0} \exp \left(-t / T_{0}\right)
$$

where $T_{0}$ is related to the intensity of noise. In figure $4 \mathrm{~d}$ we present the experimental residence time for the data of fig. 3. a. The experimental data have a characteristic time $T_{0}=1484 \mathrm{~s}=7020 T_{\text {prop }}=0.19 \tau_{\nu}$, being $\tau_{\nu}=R^{2} / \nu$ the diffusion time scale.

As a consequence of the dynamics, a natural question that arises in this problem is the response of the experiment to an external forcing. When a sinusoidal modulation is applied to the frequency of one of the propellers, the rim velocity evolves as $V_{\text {prop }}(t)=$ $V_{\text {prop }}^{0}\left(1+2 \Delta_{0} \cos (\omega t)\right)$ where $\Delta_{0}$ is the maximum frequency disymmetry.

(a)
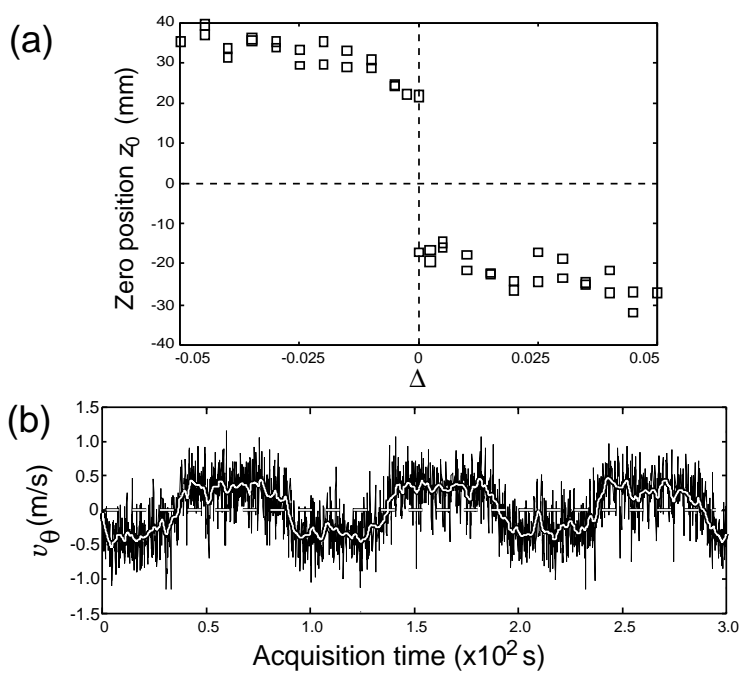

FIG. 5: (a) Position of the frontier $\left(z_{0}\right)$ varying the difference between the propellers' frequency $(\Delta)$. The system presents a bistability at $\Delta=0$. (b) Temporal evolution of the instantaneous velocity of an equatorial point near the wall, with $\Delta=0.026 \cos (0.02 \pi t), R e=2.610^{5}$. The system remains slaved to the input signal. The time scale is two orders of magnitude smaller than that of figure 3 a

A static forcing $\left(\omega=0, \Delta_{0} \neq 0\right)$ was applied to obtain the minimum amplitude needed to induce an inversion in the experiment. In Fig 5 (a) $z_{0}$ is represented vs $\Delta$. A disimmetry of only $\Delta \sim 0.0025$ is needed to make the system jump between both states. No inversions have been observed with $\Delta \neq 0$, so up to our precision and measurement times no hysteresis is found, and the system only shows a bistability in $\Delta=0$. Further investigation will determine if $T_{0}<\infty$ when $\Delta \neq 0$.

When the harmonic forcing is induced, preliminary results show how for low $f$ only a small amplitude in the input signal is needed to obtain the resonance of the system: Periodic inversions are observed with the same frequency of the forcing. Figure 3 , b shows how the system is slaved to a signal with $\Delta_{0}=0.026$ and $f=10^{-2} \mathrm{~Hz}$. In the range $10^{-2}>f / f_{\text {prop }}>10^{-3}$ it is necessary to increase $\Delta$ to observe the synchronism. For higher $f$, the system can not follow the forcing: the inertia of the flow limits the response time of the inversion $T_{\text {res }} \sim 20 \mathrm{~s}$ $=2.610^{-3} \tau_{\nu}$. Further work experimental and numerical is under run in this direction.

Conclusions - We have presented experimental evidence of a bifurcation in a turbulent system that breaks symmetries and produces slow dynamics. Two possible mirror states are equally accessible, with random (natural) or periodic (induced) inversions. The slow dynamics can be characterized using a very simple Langevin model in spite of being in a fully developed turbulence regime. Finally, when a very weak amplitude input modulation is applied to the system, a forced periodic response appears in the turbulent flow. One open question is either these natural inversions could be present in dynamo experiments for very long temporal series $\left(T_{0} \simeq 1000 \mathrm{~s}\right)$ when $\Delta=0$.

Acknowledgments - We are grateful to Jean Bragard, Iker Zuriguel and Diego Maza for fruitful discussions. This work has been supported by the Spanish government (research projects FIS2004-06596-C02-01 and UNAV0533-001) and by the University of Navarra (PIUNA program). One of us, AdlT, thanks the "Asociación de Amigos" for a post-graduate grant.

* Electronic address: admonguio@alumni.unav.es

$\dagger$ Electronic address: javier@fisica.unav.es

[1] U. Frisch, Turbulence (Cambridge Univ. Press, 1995).

[2] P. Holmes, J.L. Lumley, G. Berkooz, Turbulence, Coherent Structures, Dynamical Systems and Symmetry (Cambridge University Press, 1996).

[3] T. von Kármán, Z. Angew Math. Mech 1, 233 (1921).

[4] P. Zandbergen, D. Dijkstra, Ann. Rev. Fluid Mech. 19, 465 (1987).

[5] W. Z. Shen,J. Norensen, J. Michelsen, Phys. Fluids 18, 064102 (2006).

[6] C. Nore, L. M. Witkowski, E. Foucault, J. Pecheux, O. Daube, P. Le Quere, Phys. Fluids 18, 054102 (2006).

[7] C. Nore, L. S. Tuckerman, O. Daube, S. Xin, J. Fluid Mech. 477, 51 (2003).

[8] F. Ravelet, A. Chiffaudel, F. Daviaud, J. Leorat, Phys. Fluids 17, 117104 (2005).

[9] L. Marie, J. Burguete, F. Daviaud, J. Leorat, Eur. Phys. J B 33, 469 (2003).

[10] C. Nore, F. Moisy, L. Quartier, Phys. Fluids 17, 064103 (2005).

[11] F. Ravelet, L. Marie, A. Chiffaudel, F. Daviaud, Phys. Rev. Lett. 93, 164501 (2004).

[12] R. Monchaux et al., Phys. Rev. Lett. 98, 044502 (2007).

[13] M. Berhanu et al., Europhys Lett 77 (2007) 59001.

[14] A. Gailitis et al., Phys. Rev. Lett. 84, 4365 (2000).

[15] R. Stieglitz and U. Müller, Phys. Fluids 13561 (2001)

[16] F. Petrelis et al. Phys. Rev. Lett. 90174501 (2003)

[17] P.E. Kloeden, E. Platen , Numerical Solution of Stochastic Differential Equations (Springer, 1999).

[18] H. Kramers, Physica A 7, 284 (1940).

[19] P. Hänggi, P. Talkner, Rev. Mod. Phys. 62, 251 (1990).

[20] A. Chechkin, V. Gonchar, J. Klafter, R. Metzler, Europhys. Lett. 72, 348 (2005). 